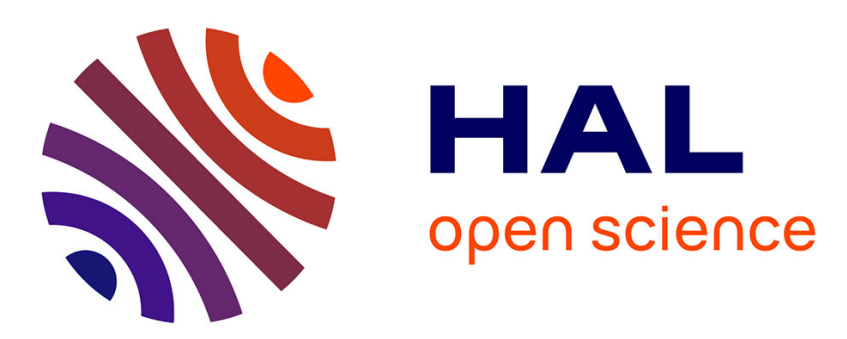

\title{
Administration de GRF ou de somatotropine chez le porc et les volailles : Effets sur les performances, la qualité des viandes et la fonction de reproduction
}

Michel Bonneau

\section{- To cite this version:}

Michel Bonneau. Administration de GRF ou de somatotropine chez le porc et les volailles: Effets sur les performances, la qualité des viandes et la fonction de reproduction. Productions Animales, 1992, 5 (4), pp.257-267. hal-00895981

\section{HAL Id: hal-00895981 \\ https://hal.science/hal-00895981}

Submitted on 1 Jan 1992

HAL is a multi-disciplinary open access archive for the deposit and dissemination of scientific research documents, whether they are published or not. The documents may come from teaching and research institutions in France or abroad, or from public or private research centers.
L'archive ouverte pluridisciplinaire HAL, est destinée au dépôt et à la diffusion de documents scientifiques de niveau recherche, publiés ou non, émanant des établissements d'enseignement et de recherche français ou étrangers, des laboratoires publics ou privés. 
INRA Prod. Anim., 1992, 5 (4), $257-267$

\section{BONNEAU}

INRA Station de Recherches porcines 35590 St Gilles

Administration de

GRF ou de

somatotropine

chez le porc et

les volailles :

Effets sur les

performances, la qualité des viandes et la fonction de reproduction

\section{L'utilisation de la somatotropine bovine (bST) en production laitière fait actuellement l'objet d'un vif débat aussi bien en Europe que sur le continent américain. L'analogue porcin de la bST, la somatotropine porcine (pST), fait également l'objet de recherches très soutenues. L'utilisation de pST modifiant considérablement l'efficacité de la croissance musculaire du porc, pourrait avoir des conséquences importantes sur la production porcine.}

\section{Résumé}

Chez des porcs nourris à volonté, l'administration de somatotropine porcine (pST) pendant la période de finition entraîne une diminution de l'ingéré alimentaire (2-22\%), une accélération de la croissance (jusqu'à $47 \%$ ) et une amélioration de l'efficacité alimentaire $(3-38 \%)$, en relation avec une réduction du dépôt de gras corporel (7-44\%). Le pourcentage de muscles dans la carcasse est augmenté de 2 à $23 \%$. En liaison avec l'augmentation du poids de certains organes (foie, coeur, etc.), le rendement à l'abattage est réduit de 1 à $4 \%$. L'administration de GRF (Growth Hormone-Releasing Factor) ou d'un analogue du GRF a les mêmes effets qualitatifs que celle de pST.

L'amplitude des effets de la pST peut varier en fonction :

1) de la dose de pST. L'ingéré alimentaire et le dépôt de gras décroissent linéairement avec la dose alors que la réponse en termes d'efficacité alimentaire ou de dépôt de muscle plafonne au delà de la dose seuil qui permet d'obtenir la vitesse de croissance maximale.

2) des modalités d'administration. Les formes d'administration lente semblent moins efficaces qu'une injection journalière.

3) de l'âge et du poids. Les effets de la pST administrée pendant la phase de croissance ou chez des porcs lourds sont similaires, qualitativement et quantitativement, à ceux observés pendant la période de finition.

4) du génotype, du sexe et de la castration. Les effets de la pST sont d'autant plus importants que le potentiel de croissance musculaire de l'animal est plus faible.

Les effets de la pST sur le pourcentage des différents types de fibres musculaires ne sont pas clairs, bien que l'on observe une augmentation générale de leur surface de section transversale. Les effets de la pST sur les caractéristiques musculaires peuvent varier fortement selon le muscle considéré. Les propriétés physiques et sensorielles de la viande ne sont généralement pas ou peu modifiées par le traitement pST. Outre l'impact sur la composition du muscle et du gras, on observe assez souvent une légère dégradation de la tendreté.

L'impact d'un traitement par la pST sur la venue en puberté des cochettes n'est pas clair. Il semble bien cependant que, s'il devait y avoir un effet négatif, celui-ci soit facilement réversible, dès l'arrêt du traitement. L'administration de pST pendant la gestation n'a aucun effet sur la portée. L'intérêt d'un traitement par la pST pendant la lactation reste controversé.

Bien que la somatotropine semble être impliquée dans la régulation de la croissance chez les oiseaux, l'administration de GRF ou de somatotropine n'a que des effets transitoires (ou même, le plus souvent, aucun effet) sur les performances de croissance et la composition des carcasses de poulet...
Cet article passe en revue les données expérimentales concernant les effets de l'administration exogène de GRF (Growth HormoneReleasing Factor) ou de somatotropine (ST) sur les performances de croissance, la composition des carcasses, la qualité des viandes et la fonction de reproduction chez les porcs et les volailles.

La ST, aussi appelée hormone de croissance, est sécrétée par l'hypophyse sous la dépendance de deux hormones d'origine hypothalamique. Le GRF stimule alors que la somatostatine (ou SRIF, Somatotropin ReleaseInhibiting Factor) inhibe la sécrétion hypophysaire de ST. La ST a des actions multiples, somatogéniques et métaboliques. Elle agit sur les tissus cibles, soit directement, soit via l'action des IGFs (Insulin Like Growth Factors, ou somatomédines) dont elle stimule la synthèse, principalement au niveau du foie mais aussi dans de nombreux autres sites, y compris les tissus cibles de la ST eux-mêmes.

\section{1 / Effets de l'administration de pST ou de GRF sur les performances de croissance du porc}

Sauf spécification particulière, les résultats présentés dans cet article ont été obtenus avec des injections journalières des hormones. Peu de données ont été publiées sur l'efficacité des 
implants (Knight et al 1988, 1989, 1990 , Baile et al 1989, Becker et al 1989).

\section{1 / Effets de la somatotropine porcine (pST)}

Les premiers essais visant à mettre en évidence les effets de l'administration de pST sur les performances du porc ont été réalisés avec des extraits hypophysaires imparfaitement purifiés (Turman et Andrews 1955, Henricson et Ullberg 1960, Lind et al 1968, Machlin 1972). Les progrès de la biochimie ont conduit à de meilleures préparations hypophysaires au cours des années 1980. Mais, au même moment, le GRF a été découvert et la pST d'origine recombinante est devenue disponible. Cette pST recombinante a les mêmes effets biologiques et zootechniques que celle d'origine hypophysaire (Ivy et al 1986, Evock et al 1988).

Une sélection de résultats sur l'influence de l'administration de pST sur les performances de croissance et la composition des carcasses de porcs en finition alimentés à volonté est présentée au tableau 1 .

Tableau 1. Effets de l'administration de pST sur les performances de croissance et la composition des carcasses de porcs en finition alimentés à volonté (données exprimées en \% des témoins).

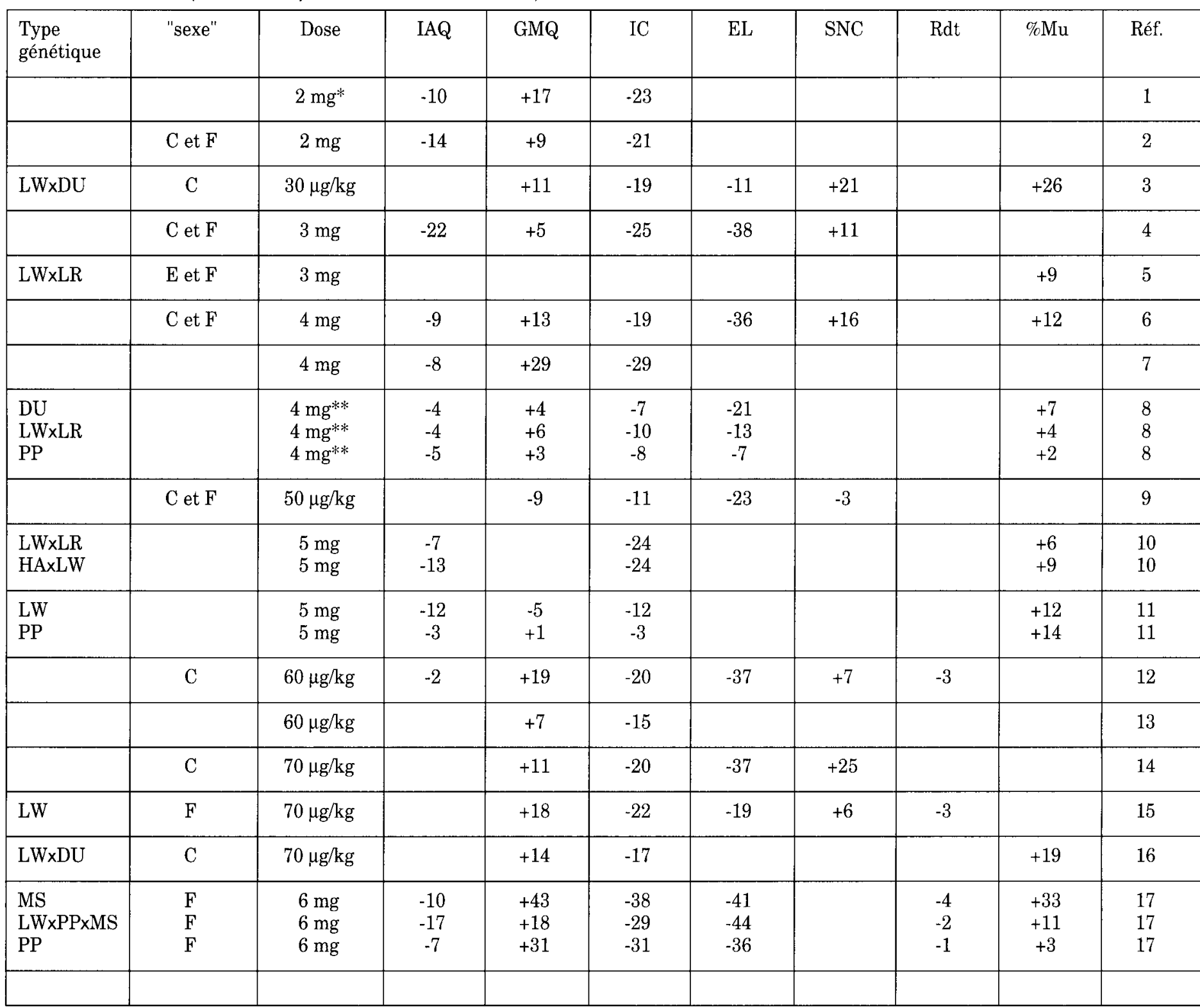

Race $:$ DU = Duroc, $\mathrm{HA}=$ Hampshire, $\mathrm{LR}=$ Landrace, $\mathrm{LW}=$ Large-White, $\mathrm{MS}=$ Meishan, $\mathrm{PP}=$ Piétrain .

"Sexe" : $\mathrm{E}=$ mâles entiers, $\mathrm{C}=$ mâles castrés, $\mathrm{F}=$ femelles.

Dose : dose journalière.

$\mathrm{IAQ}=$ Ingéré Alimentaire Quotidien, GMQ = Gain Moyen Quotidien, IC = Indice de Consommation, EL = épaisseur de Lard, SNC = Surface de la Noix de Côtelette, Rdt = Rendement à l'abattage, \%Mu = Pourcentage de Muscles de la carcasse.

*sous forme d'implant, ${ }^{* *}$ en injection bihebdomadaire de $14 \mathrm{mg}$

Réf.: 1 = Knight et al 1988, 2 = Azain et al 1989, 3 = Etherton et al 1986, 4 = Bechtel et al 1988, 5 = Baile et al 1990, 6 = Skaggs et al 1989c, $7=$ Goodbank et al 1988, 8 = Kanis et al 1990, 9 = Smith et al 1987, $10=$ Nossaman et al 1989, 1991, $11=$ Fowler et Kanis 1988, $12=$ Trenkle 1988, $13=$ Jones et al 1989,

$14=$ Bark et al 1989, 15 = Bryan et al 1989a, 16 = Etherton et al 1987, 17 = Bidanel et al 1991, 18 = Campbell et Taverner 1988 . 
En dépit d'une réduction significative de l'ingéré alimentaire quotidien, les porcs traités par la pST présentent une croissance plus rapide et une bien meilleure efficacité alimentaire. L'adiposité des carcasses est fortement réduite avec une augmentation concomitante de la proportion de muscles. De façon plus générale, la pST favorise le développement de tous les tissus riches en protéines (muscle, peau et os) au détriment du gras (Bonneau et al 1989, Bark et al 1990, Caperna et al 1990, Thiel et al 1990b).

Le rendement à l'abattage chute de 1 à $4 \%$ après administration de $\mathrm{pST}$. Cette décroissance s'explique pour partie par le développement accru de certains organes. Les poids de foie, de coeur et de rein sont accrus de $4-30 \%, 2-22 \%$ et 15-52\%, respectivement (Grebner et al 1987, Bechtel et al 1988, Evock et al 1988, Kanis et al 1988b, Trenkle 1988, Bonneau et al 1989, Bryan et al 1989a). Des résultats préliminaires (Bidanel et al, non publiés) suggèrent qu'une augmentation du volume sanguin et des contenus intestinaux pourraient aussi avoir une part de responsabilité dans la chute de rendement chez les porcs traités par la pST.

\section{2 / Effets du GRF}

Des injections quotidiennes ou triquotidiennes de GRF, ou d'un analogue du GRF, augmentent les taux circulants de pST, de façon dose dépendante. Cette stimulation est observée tout au long de la période d'administration, sans que ne se manifeste aucune désensibilisation des cellules somatotrophes (Dubreuil et al 1990a, 1990b). Les effets du GRF ou d'un analogue du GRF sont qualitativement similaires à ceux de la pST (tableau 2). Cependant, à dose administrée égale, l'amélioration des performances est sensiblement plus faible, avec le GRF qu'avec la pST (Etherton et al 1986, Johnson et al 1989).
Comme la pST, le GRF ou son analogue accélèrent le développement de certains organes (Dubreuil et al 1990b) et de tous les tissus riches en protéines (muscle, peau, os) au détriment du gras (Pommier et al 1990).

\section{3 / Conclusion}

L'administration de pST ou de GRF améliore de facon spectaculaire aussi bien la vitesse que l'efficacité de la croissance des tissus maigres au détriment des dépôts adipeux, ce qui montre que les hormones somatotropes sont un facteur limitant important des performances chez le porc. Ceci peut être relié au fait que les niveaux circulants endogènes de pST tombent à des valeurs faibles chez le porc en croissance-finition, par rapport aux concentrations observées pendant la période néonatale (Klindt et Stone 1984, Scanes et al 1987, Louveau et al 1990).

\section{2 / Facteurs de variation de la réponse à l'administration de pST chez le porc}

Parmi les facteurs pris en considération, on distinguera ceux qui sont liés à la pST ellemême (dose, forme et schémas d'administration) de ceux qui dépendent de l'animal (âge et poids, génotype, sexe et castration) ou de l'environnement (conditions nutritionnelles). On s'intéressera aussi aux interactions possibles entre la pST et les $\beta$-agonistes.

\section{1 / Modalités d'administration}

La dose de pST injectée peut être soit constante (elle est alors exprimée en mg par jour) soit adaptée au poids croissant de l'ani-
Les porcs traités parla pST présentent une croissance plus rapide et une bien meilleure efficacité alimentaire.

Tableau 2. Effets de l'administration de GRF sur les performances de croissance et la composition des carcasses de porcs en finition alimentés à volonté (données exprimées en \% des témoins).

\begin{tabular}{|c|c|c|c|c|c|c|c|c|c|c|}
\hline GRF & Dose & NI & IAQ & GMQ & IC & EL & $\mathrm{SNC}$ & $\mathrm{Rdt}$ & $\% \mathrm{Mu}$ & Réf. \\
\hline GRF1-44 & 30 & 1 & & +6 & -9 & & +13 & & & 1 \\
\hline GRF1-44 & 40 & 2 & -8 & +14 & -19 & -22 & +12 & & & 2 \\
\hline analogue & 5 & 3 & -8 & +3 & -11 & -7 & +2 & -1 & +3 & 4 \\
\hline analogue & 10 & 3 & -6 & +12 & -16 & -24 & +4 & -3 & +5 & 4 \\
\hline analogue & 20 & 3 & -21 & +7 & -26 & -32 & +13 & -2 & +6 & 4 \\
\hline analogue & 10 & 1 & -10 & -1 & -11 & -16 & +10 & -1 & +5 & 4 \\
\hline
\end{tabular}

Dose : dose journalière. $(\mu \mathrm{g} / \mathrm{kg} / \mathrm{j})$.

$\mathrm{IAQ}=$ Ingéré Alimentaire Quotidien, GMQ = Gain Moyen Quotidien, IC = Indice de Consommation, EL = épaisseur de Lard, SNC = Surface de la Noix de Côtelette,

Rdt $=$ Rendement à l'abattage, $\% \mathrm{Mu}=$ Pourcentage de Muscles de la carcasse.

Réf. : 1 = Etherton et al 1986, $2=$ Johnson et al 1989, $3=$ Dubreuil et al 1990c, $4=$ Dubreuil et al 1990b.

$\mathrm{NI}=$ Nombre d'Injections quotidiennes 
mal (elle est alors exprimée en $\mu \mathrm{g} / \mathrm{kg} / \mathrm{jour}$ ). La dose qui donne l'effet maximum varie en fonction du critère considéré (figure 1). L'ingéré alimentaire quotidien décroît linéairement avec la dose alors que l'efficacité alimentaire plafonne au delà de 80-100 $\mu \mathrm{g} / \mathrm{kg} / \mathrm{j}$. En conséquence, la réponse en terme de vitesse de croissance est de type quadratique. La dose qui maximise le gain GMQ peut varier en fonction des conditions expérimentales, de $3 \mathrm{mg} / \mathrm{j}$ (Darden et al 1990, Fitzner et al 1990) à 6 $\mathrm{mg} / \mathrm{j}$ ou $60-70 \mu \mathrm{g} / \mathrm{kg} / \mathrm{j}$ (Boyd et al 1986, Mc Laren et al 1987, Evock et al 1988). L'adiposité des carcasses diminue linéairement avec la dose, alors que le pourcentage de muscles augmente de façon quadratique (Boyd et al 1986, McNamara et al 1990, Yen et al 1990). Cependant, Thiel et al (1990b) ont observé une augmentation linéaire du pourcentage de muscles avec des doses de pST allant de 50 à $200 \mu \mathrm{g} / \mathrm{kg} / \mathrm{j}$.

Il y a peu d'information disponible sur l'efficacité comparée d'une injection journalière et d'une administration continue de pST. Des observations préliminaires de Knight et al (1988) semblent indiquer que la pST administrée en continu est moins efficace qu'une injection quotidienne.

Figure 1. Effet de la dose de pST sur les performances et la composition des carcasses de porcs (données exprimées en \% des témoins).
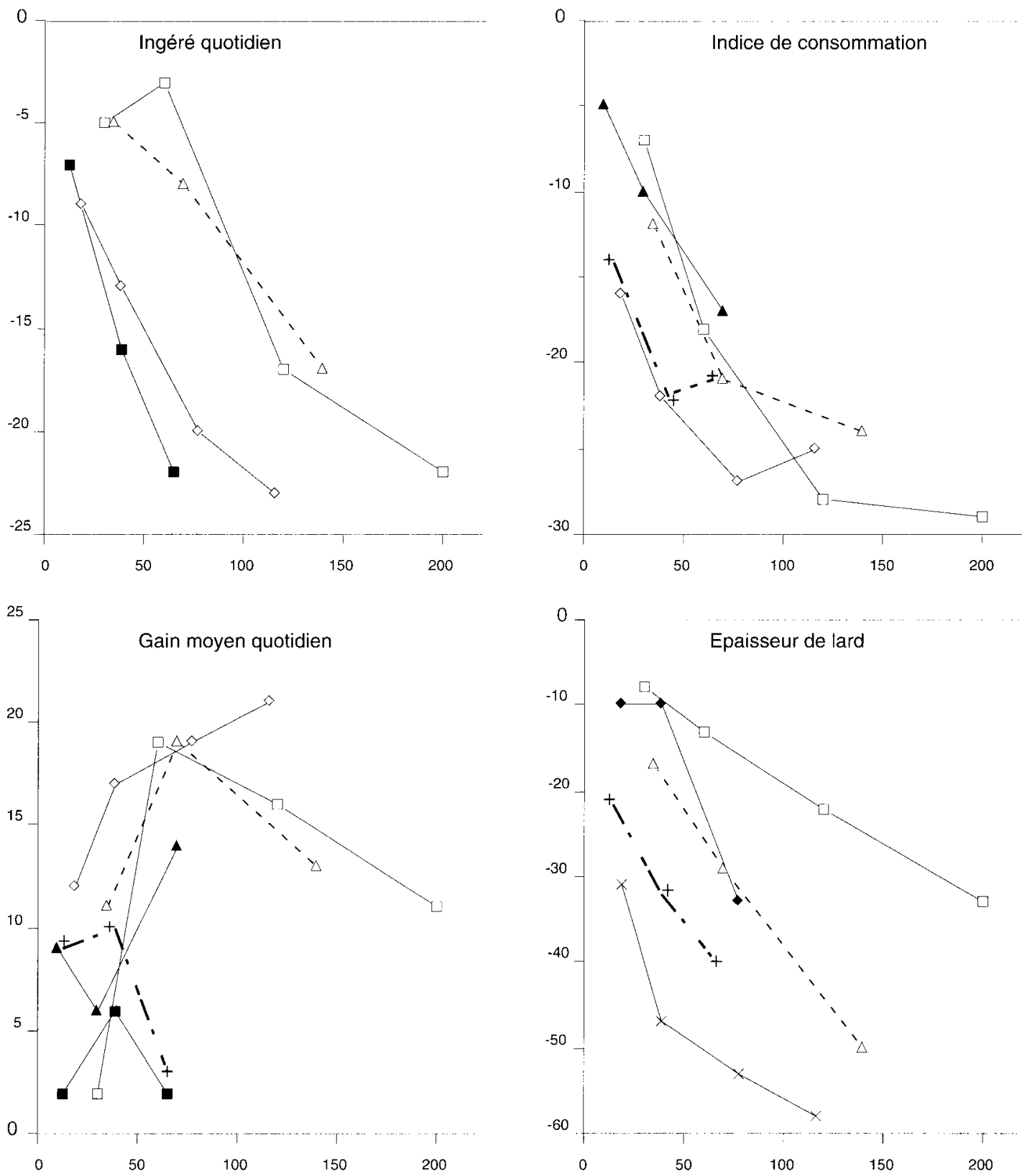

dose de pST $(\mu \mathrm{g} / \mathrm{kg} / \mathrm{j})$

Rebhun et Etherton 1985

Boyd et al 1986
- Demeyer et a/ 1988

Mc Laren et al 1987 et Grebner et al 1987 $\triangle \quad$ Evock et al 1988

- Mc Laren et al 1990
Fitzner et al 1990

+ Darden et al 1990 et Baldwin et al 1990 
Van Vlissingen et al (1990a) n'ont mis en évidence que des différences mineures pour les effets sur les performances et la qualité des carcasses entre trois schémas d'administration de la pST : dose constante $(4 \mathrm{mg} / \mathrm{j})$, croissante (de 2 à $6 \mathrm{mg} / \mathrm{j}$ ) ou décroissante (de 6 à 2 $\mathrm{mg} / \mathrm{j}$ ). Comme on pouvait s'y attendre, l'amélioration de la vitesse de croissance et de la surface de noix de côtelette est plus importante avec des injections quotidiennes pratiquées en continu qu'avec des injections quotidiennes pratiquées une semaine sur deux (Bryan et al 1990b).

\section{2 / Age et poids}

La période de finition, au cours de laquelle la croissance musculaire atteint sa valeur maximale, alors que le développement du tissu adipeux s'accélère, est en général considérée comme la période la plus favorable à la manifestation des effets de la pST chez le porc. Mais la pST peut aussi être efficace à d'autres périodes.

Un traitement par la pST pendant la période de croissance (de 25-30 à 50-60 kg) a des effets similaires à ceux observés chez des porcs plus lourds. L'administration de $100 \mu \mathrm{g} / \mathrm{kg} / \mathrm{j}$ accroît la rétention protéique de $35-50 \%$ et réduit le dépôt adipeux de 26-32 \% (Campbell et al 1988a).

Les effets de la pST s'observent aussi chez des animaux traités au-delà de $100 \mathrm{~kg}$ de poids vif. L'amélioration des performances est similaire (Jones et al 1989, Crenshaw et al 1990, Shoup et al 1990) ou même plus forte (Kanis et al 1990) que celle observée chez des porcs traités en finition.

Selon Campbell et al (1989b), des animaux traités par la pST pendant la phase de croissance présentent encore des performances améliorées, pendant la période de finition, longtemps après l'arrêt du traitement. Mais la réalité de cet effet rémanent est controversée (Smith et al 1989).

\section{3 / Génotype}

Dans tous les génotypes de porc étudiés jusqu'à présent, les performances répondent favorablement au traitement par la pST. Dans le cas de génotypes conventionnels (races ou croisements utilisés dans la pratique courante de la production), on n'observe pas d'interaction entre le traitement par la pST et le génotype (Bark et al 1990, Shoup et al 1990). Les effets de la pST sur les performances sont similaires dans des lignées de porcs sélectionnées pour le maigre ou pour le gras (Campbell et Taverner 1988, Bark et al 1989). Les porcs des trois génotypes possibles au locus de la sensibilité à l'halothane (NN : Normal/Normal ; Nn : Normal/sensible ; nn : sensible/sensible) répondent aussi de façon similaire au traite- ment par la pST. On note cependant que l'amélioration de l'efficacité alimentaire est plus faible chez les porcs nn que chez les NN (Skaggs et al 1989a, 1989b).

Par contre, une interaction génotype $\mathrm{x}$ traitement est clairement démontrée pour les effets de la pST lorsque l'on compare des porcs gras à des maigres (Yen et al 1990), des animaux gras à des hypermusclés (Bidanel et al 1991) ou des porcs conventionnels à des hypermusclés (Kanis et al 1990, Bidanel et al 1991). L'amélioration des performances est particulièrement spectaculaire chez les porcs de race Meishan, animaux gras à croissance lente, aussi bien en race pure qu'en croisement avec des races conventionnelles (Fung et Qi 1988, McLoughlin et al 1989, Van Der Steen et al 1989, Prunier et al 1990, Bidanel et al 1991). Dans les races très musclées, comme le Piétrain ou le Landrace Belge, le gras souscutané disparaît presque, alors que le pourcentage de muscle de la carcasse n'est que légèrement amélioré (Demeyer et al 1988, Bonneau et al 1990a, Kanis et al 1990). En règle générale, on peut considérer que l'amélioration relative des performances apportée par le traitement pST est inversement proportionnelle au potentiel de l'animal pour la croissance du tissu maigre. Cependant les effets de la pST sont encore significatifs dans les races les plus maigres (Kanis et al 1990, Bonneau et al 1990a, Noblet et al 1992).

\section{4 / Sexe et castration}

Les effets d'une forte dose de pST (100 $\mu \mathrm{g} / \mathrm{kg} / \mathrm{j}$ ) sont plus prononcés chez les mâles castrés que chez les femelles et chez les femelles que chez les mâles entiers, si bien que les différences de performances entre types sexuels sont quasiment gommées chez les porcs traités par la pST (Campbell et al 1989a). Avec des doses de pST plus faibles ( 3 ou $4 \mathrm{mg} / \mathrm{j}$ ), les mâles castrés présentent encore une réponse à la pST plus importante, mais la supériorité des femelles, plus maigres que les castrats, se maintient chez les animaux traités (Bonneau et al 1989, Kanis et al 1990).

\section{5 / Conditions nutritionnelles}

\section{Niveau d'alimentation et apports énergétiques}

Les résultats présentés précédemment ont été obtenus chez des animaux nourris à volonté. Le tableau 3 rassemble les résultats obtenus quand la consommation d'aliment est égalisée entre animaux témoins et traités. Lorsqu'une restriction alimentaire modérée est appliquée (80-85\% du niveau à volonté), l'écart se creuse entre animaux témoins et porcs traités, les performances de ces derniers étant moins affectées par la restriction.

Selon Verstegen et al (1988), les besoins énergétiques d'entretien du porc traité à la
Les effets de la pST s'observent pendant la croissance mais aussi au-delà de $100 \mathrm{~kg}$ de poids vif. 
Tableau 3. Effets de l'administration de pST sur les performances de croissance et la composition des carcasses de porcs recevant une alimentation égalisée (données exprimées en \% des témoins).

\begin{tabular}{|l|c|c|c|c|c|c|c|c|c|c|}
\hline $\begin{array}{l}\text { Type } \\
\text { génétique }\end{array}$ & "sexe" & Dose & $\begin{array}{c}\text { Niveau } \\
\text { Alim. }\end{array}$ & GMQ & IC & EL & SNC & Rdt & $\%$ Mu & Réf. \\
\hline & C et F & $3 \mathrm{mg}$ & 100 & +37 & -30 & -23 & +11 & & & 1 \\
LW & C et F & $3 \mathrm{mg}$ & 80 & +56 & -36 & -29 & +8 & & & 1 \\
LW & $\mathrm{C}$ & $3 \mathrm{mg}$ & 100 & +32 & -25 & -30 & +5 & -2 & +9 & 2 \\
LWXLR & $\mathrm{F}$ & $3 \mathrm{mg}$ & 100 & +21 & -21 & -19 & +9 & -2 & +7 & 2 \\
LWxLR & & $5 \mathrm{mg}$ & 93 & & -21 & & & & +5 & 3 \\
LWxLR & & $5 \mathrm{mg}$ & 86 & & -23 & & & & & \\
HAxLW & & $5 \mathrm{mg}$ & 80 & & -33 & & & & +12 & 3 \\
HAxLW & & $5 \mathrm{mg}$ & 93 & & -21 & & & & +9 & 3 \\
HAxLW & & $5 \mathrm{mg}$ & 86 & & -29 & & & & +8 & 3 \\
\hline
\end{tabular}

Dose : dose journalière.

Niveau alim. : niveau d'alimentation exprimé en $\%$ du niveau de l'ad libitum de l'animal traité par la pST

GMQ = Gain Moyen Quotidien, IC = Indice de Consommation, EL = Epaisseur de Lard, SNC = Surface de la Noix de Côtelette, Rdt = Rendement à l'abattage,

$\% \mathrm{Mu}=$ Pourcentage de muscles de la carcasse.

Réf. : = 1 Bechtel et al 1988, 2 = Bonneau et al 1989, 3 = Nossaman et al 1989.

Les performances des porcs traités à la pST sont moins affectées par une restriction alimentaire.
pST seraient augmentés d'environ $10 \%$. Les besoins énergétiques de production diminuent du fait de la réduction du dépôt de lipides corporels, en dépit de l'accroissement de la rétention de protéines dont le rendement énergétique est faible. En relation avec l'amélioration spectaculaire de l'efficacité alimentaire, les besoins énergétiques globaux de l'animal sont fortement abaissés (Noblet et al 1992).

\section{Apports de protéines}

Une teneur en protéines trop faible de l'aliment limite fortement la réponse de l'animal au traitement par la pST (Campbell et al 1988b, Anderson et al 1989, Fowler et al 1989, Smith et al 1989a). L'augmentation de la quantité de protéines fixées (Huisman et al 1988, Anderson et al 1989, Ender et al 1989a) devrait accroître le besoin journalier de protéines, mais cet effet pourrait être contrebalancé par un meilleur rendement de fixation de l'azote absorbé (Noblet et al 1992). Compte tenu de la réduction de l'appétit des animaux, il semble vraisemblable que le besoin, exprimé en taux de protéines dans l'aliment, soit accru.

\section{6 / Interaction avec les $\beta$-agonistes}

Sous réserve de conditions nutritionelles satisfaisantes, les effets de la pST et des $\beta$-agonistes semblent être additifs chez le porc (Anderson et al 1989, Jones et al 1989).

\section{3 / Qualité de la viande des porcs traités par la pST}

\section{1 / Composition du muscle}

\section{Composition chimique du muscle}

La pST entraîne de légères modifications de la composition chimique du muscle qui est plus riche en protéines et en eau, mais plus pauvre en lipides (Beerman et al 1988, Fowler et Kanis 1988, Prusa 1988, Bonneau et al 1989). Cette réduction de la teneur en lipides intramusculaires, qui n'est pas toujours observée (Novakofski 1987, Demeyer et al 1988, Kanis et al 1988c), pourrait avoir des conséquences défavorables sur les propriétés sensorielles des viandes. Prusa (1988) rapporte que la teneur en cholestérol du muscle long dorsal est légèrement augmentée par le traitement à la pST, dans des limites qui, selon l'auteur, devraient être "sans grande conséquence pratique pour les personnes soucieuses de contrôler leur niveau d'ingestion de cholestérol".

\section{Types de fibres musculaires}

Les effets du traitement par la pST sur le pourcentage et la taille des différents types de fibres musculaires restent controversés. Dans les muscles Longissimus dorsi ou Semitendinosus, la pST augmente la taille des fibres (Beerman et al 1987, Solomon et al 1988, 1990, Lefaucheur et al, non publié). Cependant, dans le même muscle, Whipple et al 
(1989) ne trouvent aucune différence de taille de fibre entre animaux traités et témoins. La proportion des différents types de fibres musculaires dans le Longissimus dorsi peut être inchangée (Solomon et al 1988, Lefaucheur et al, non publié) ou modifiée dans le sens d'une augmentation (Whipple et al 1989) ou d'une diminution (Solomon et al 1990) du pourcentage de fibres blanches. Dans un autre muscle blanc, le Semimembranosus, ni le pourcentage des différents types de fibres, ni leur taille ne sont significativement affectés par le traitement pST (Whipple et al 1989). Dans le muscle rouge Semispinalis, la proportion de fibres de type IIB et la taille de tous les types de fibres sont accrues par l'administration de pST (Lefaucheur et al, non publié). Dans ce même muscle, l'activité de la lactate déshydrogénase est augmentée alors que celles de la citrate synthase et de la $\beta$-hydroxy-acyl-CoA-déshydrogénase sont réduites par le traitement pST (Lefaucheur et al, non publié). En résumé, la pST semble accroître la taille des fibres avec un effet encore controversé sur le pourcentage des différents types de fibres dans les muscles blancs. Le métabolisme des muscles rouges est modifié vers une réduction de la voie oxydative au profit de la voie glycolytique, en relation avec l'augmentation du pourcentage des fibres de type IIB. Le fait que les effets de la pST puissent varier selon le type de muscle est confïmé par les observations d'Evock et al (1990) : la pST augmente le contenu en ARN et le rapport $A R N / A D N$ dans le Longissimus dorsi alors que le contenu en ADN est accru dans le muscle Semimembranosus.

\section{2 / Composition du gras}

Comme dans tous les cas où le dépôt adipeux est réduit, la composition en acides gras des graisses est modifiée par le traitement pST. Le degré d'insaturation augmente, particulièrement $d u$ fait d'un accroissement important de la teneur en acide linoléique C18:2 (Ender et al 1989b, Mourot et al 1991). L'augmentation du taux d'insaturation des graisses est une caractéristique favorable sur le plan diététique, mais défavorable du point de vue technologique.

\section{3 / Caractéristiques physiques des viandes}

La chute du pH après l'abattage est en moyenne peu affectée par le traitement à la pST (Bonneau et al 1989, Ender et al 1989, Hagen et al 1990a). Le $\mathrm{pH}$ mesuré 45 minutes ou 24 heures après l'abattage peut cependant être plus élevé (Demeyer et al 1988, Skaggs et al 1989a, Mourot et al 1990). De façon similaire, une tendance à des $\mathrm{pH}$ plus élevés a été observée chez des animaux traités par un analogue du GRF (Pommier et al 1990).

Les mesures physiques de la qualité des viandes (force de cisaillement, pertes d'égouttage, pertes de cuisson, couleur) ne sont dans l'ensemble pas modifiées par le traitement à la
pST (Beerman et al 1988, Demeyer et al 1988, Kanis et al 1988c, Prusa 1988, Ender et al 1989, Gardner et al 1990, Mourot et al 1990, Williams et al 1990). Cependant, Novakofski (1987) ainsi que Williams et al (1990) observent que la force de cisaillement est augmentée dans les muscles des porcs traités par la pST.

\section{4 / Caractéristiques sensorielles des viandes}

Les caractéristiques sensorielles des viandes, appréciées par un jury de dégustation, ne sont pour l'essentiel pas modifiées (Novakofski 1987, Beerman et al 1988, Demeyer et al 1988, Kanis et al 1988c, Prusa 1988, Hagen et al 1990a, Thiel et al 1990a, Williams et al 1990). Cependant, on a pu observer des réductions légères mais significatives de la tendreté (Beermann et al 1988 , Evock et al 1988, Prusa 1988, Boles et al 1990, Thiel et al 1990a, Williams et al 1990), de la jutosité (Beermann et al 1988) ou de la flaveur (Prusa 1988). La détérioration de la tendreté pourrait provenir de l'augmentation de la teneur en collagène (Caperna et al 1990). Mais le collagène exprimé en \% des protéines totales aussi bien que son degré de solubilité ne sont pas modifiés par le traitement pST. Ces effets défavorables de la pST sur les propriétés sensorielles des viandes sont surtout observées avec de fortes doses de pST ; on considère généralement que les incidences pratiques de ces observations sont peu importantes.

Prusa et al (1990), utilisant un jury large de consommateurs, rapporte une meilleure acceptabilité pour les viandes provenant de porcs traités par la pST que pour celles des animaux témoins.

Le traitement par la pST ne semble pas modifier les propriétés technologiques des viandes ni les qualités sensorielles de saucisses de Francfort (Reagan et al 1990) ou de jambons cuits (Kuecker et al 1990).

\section{4 / Effets de l'administration de pST sur la fonction de reproduction}

\section{1 / pST et développement de l'activité sexuelle}

La réduction du tissu adipeux et les bouleversements endocriniens induits par la pST sont susceptibles d'avoir des répercussions sur le fonctionnement de la fonction de reproduction (Aherne et Kirkwood 1985, Kirkwood et al 1989). La maturation des oocytes est accélérée (Hagen et Graboski 1990). La sécrétion de progestérone par les follicules ovariens est modifiée (Bryan et al 1988, 1989a, 1989b), mais le contenu en progestérone du fluide folliculaire

\section{L'administration de pST diminue l'adiposité des carcasses et modifie la composition du gras.}


n'est pas altéré (Spicer et al 1990). Le nombre de sites de liaison pour hCG est réduit dans les cellules de la granulosa (Spicer et al 1990) et augmenté dans le tissu lutéal (Kirkwood et al $1990 \mathrm{~b}$ ). La réponse des ovaires à une stimulation par PMSG/hCG est perturbée et l'amplitude du pic de LH induit par une injection d'oestrogènes est diminuée (Kirkwood et al 1989a, 1990a)

L'influence d'un traitement par la pST sur l'apparition de la puberté chez les jeunes truies prépubères n'est pas claire. Bryan et al (1989a) observent que des cochettes traitées atteignent la puberté en moins grand nombre et plus tard que les témoins. Cependant la plupart des études ne constatent aucun effet sur l'âge à la puberté de truies traitées par des doses même fortes de pST (Andres et al 1989, Kirkwood et al 1989b, Bidanel et al 1990, Prunier et al 1990). Dans le cas des truies restées impubères, le développement du tractus génital ne semble pas affecté par le traitement par la pST (Bryan et al 1988, 1989b, 1990b, Terlouw et al 1989, Prunier et al 1990). Cependant, Hagen et al (1990b) ainsi que Bryan et al (1990b) observent une augmentation significative du poids et de la longueur de l'utérus chez des cochettes recevant $5 \mathrm{mg}$ de pST par jour pendant 20 ou 40 jours.

L'activité sexuelle semble revenir très rapidement à la normale après l'arrêt de l'administration de pST. Les truies traitées répondent de façon similaire ou même meilleure au stimulus constitué par le passage quotidien d'un verrat (Day et al 1988, Bryan et al 1990). De plus, la durée du premier cycle, le taux d'ovulation, le pourcentage de femelles gestantes et la mortalité embryonnaire ne sont pas altérés (Day et al 1988, Andres et al 1989, 1990, Kirkwood et al 1989b, 1989c). En résumé, les effets éventuels de l'administration chronique de pST sur la venue en puberté des cochettes semblent transitoires.

Il n'y a guère d'information disponible concernant les effets de l'administration de pST sur le développement sexuel des verrats. La pST n'a pas d'effet significatif sur le développement de l'appareil génital (Hagen et al 1990a, Bonneau et al, sous presse) ou sur les capacités de synthèse testiculaire de stéroides (Bonneau et al, sous presse). Cependant la teneur en androsténone (stéroïde d'origine testiculaire, responsable des odeurs sexuelles) des graisses est plus faible après traitement par la pST (Bonneau et al, sous presse).

\section{2 / Administration de pST aux truies en gestation ou en lactation}

L'administration de pST à des truies pendant les 2 ou 3 dernières semaines de la gestation n'a aucun effet significatif sur le poids des porcelets à la naissance ou au sevrage, ni sur leur taux de survie (Kveragas et al 1986 , Baile et al 1989). Cependant, Kveragas et al (1986) signalent que les porcelets nés de mères traitées ont de plus grandes réserves de glycogène et de lipides à la naissance, et que leur glycémie évolue plus favorablement au cours d'un jeûne.

Selon Harkins et al (1989), l'injection de pST pendant la lactation entraine une augmentation importante de la production laitière et des quantités de matières utiles exportées dans le lait, la composition du lait restant inchangée. Le poids des porcelets au sevrage est ainsi accru de $6 \%$ lorsque les mères sont traitées à la pST. L'accroissement de la production laitière, conjuguée à une diminution de l'ingéré alimentaire, entraîne une mobilisation plus importante des réserves corporelles chez les truies traitées. Les autres auteurs ne confirment pas cet effet stimulant de la pST sur la production laitière. Mais il faut souligner que leurs conditions expérimentales étaient très défavorables à l'obtention d'un résultat positif, soit en raison de conditions d'environnement climatique très dures, entraînant une forte mortalité (Cromwell et al 1989a), soit du fait d'une fréquence beaucoup trop faible d'injection de la pST (1 fois par semaine, Crenshaw et al 1989, Cromwell et al $1989 \mathrm{~b})$.

\section{5 / Effets de la ST chez les volailles}

Comme chez les mammifères, la ST semble impliquée dans la régulation de la croissance des volailles. La ST a une action lipolytique et antilipogénique chez les oiseaux (John et al 1973, Foltzer et al 1975, Foltzer et Miahle 1976, Harvey et al 1977, Campbell et Scanes 1986). L'administration d'antisérum antiST (Scanes et al 1977) ou l'hypophysectomie (Nalbandov et Card 1943, King 1969, Scanes et al 1986) entraînent une réduction de la vitesse de croissance. Cependant, les effets de l'hypophysectomie ne sont que partiellement compensés par l'administration de ST de poulet (cST) ou de ST mammalienne, alors que la croissance est totalement rétablie par injection de T3 (Marsh et al 1984, Scanes et al 1986). De plus, on n'a jamais mis en évidence de relation positive entre le potentiel de croissance et la sécrétion de cST. Les poulets nains présentent des niveaux circulants de cST plus élevés que les poulets normaux (Harvey et al 1984, Lilburn et al 1986, Huybrechts et al 1987). De même, les niveaux sanguins de cST sont plus élevés dans les souches à croissance lente que dans celles à croissance rapide (Burke et Marks 1982, Stewart et Washburn 1983, Lilburn et al 1986, Goddard et al 1988). Cependant, les niveaux d'IGF-1 sont plus forts chez les poulets normaux que chez les nains (Huybrechts et al 1987), en relation avec un nombre plus important de récepteurs hépatiques à la cST (Leung et al 1987, Kuhn et al 1989).

Il a été montré que l'immunoneutralisation de la somatostatine augmente la vitesse de 
croissance et réduit le gras abdominal chez le poulet (Spencer et al 1986, Buonomo et al 1987). Cependant, comme ni les niveaux de cST ni ceux d'IGF-1 n'étaient augmentés chez les oiseaux immunisés, il se peut très bien que l'amélioration des performances obtenue par immunisation anti somatostatine résulte de mécanismes sans relation avec l'axe somatotrope.

\section{1 / Effets des ST d'origine mammalienne}

Dans les études les plus anciennes, des préparations relativement impures de ST ovine (oST) ou bovine (bST) n'ont pas stimulé la croissance des poulets (Eaton et al 1955, Libby et al 1955, Glick 1960). Des préparations plus pures de bST ou d'oST ou encore de la bST recombinante (r-bST) n'ont aucun effet sur la vitesse de croissance de poulets normaux (Scanes et al 1975, 1984, Tojo et al 1979, Marsh et al 1984). Cependant, il a été observé une amélioration de la croissance chez des poulets traités entre 6 et 12 semaines d'âge par un digesta trypsique de bST (Myers et Peterson 1974) ou chez des embryons de poulet recevant une préparation de bST (Hsieh et al 1952). L'administration de $r$-bST à très forte dose $(0,5$ ou $2,5 \mathrm{mg}$ par $\mathrm{kg}$ poids et par jour $)$ chez des poulets de chair femelles entre 4 et 6 semaines d'âge accélère la croissance et augmente l'ingéré alimentaire pendant la première semaine du traitement (Buonomo et Baile 1988). Mais la r-bST n'a eu aucun effet sur les performances pendant la deuxième semaine, pendant laquelle des titres élevés en anticorps ont été mesurés dans le sang. La r-bST n'a eu aucun effet sur la composition des carcasses à la fin des 2 semaines de traitement.

\section{2 / Effets de la somatotropine de poulet (cST)}

Des préparations purifiées ou semipurifiées de cST administrées sur de courtes périodes (4-9 jours) stimulent la croissance de jeunes poulets (Tojo et al 1979, Scanes et al 1986). Leung et al (1986) observent une stimulation transitoire de la croissance corporelle chez des coquelets de 4 semaines recevant une dose journalière de $5,10 \mathrm{ou} 50 \mu \mathrm{g}$ de cST d'origine hypophysaire (p-cST) pendant 2 semaines. Cependant, les différences entre animaux traités et témoins n'étaient pas significatives à la fin du traitement. Une infusion continue de p-cST $(20 \mu \mathrm{g} / \mathrm{kg} / \mathrm{jour})$ chez des poulettes de $2-5$ semaines n'a eu aucun effet sur l'ingéré alimentaire, le gain de poids ou la composition corporelle (Cravener et al 1989). Les études précédentes sur les effets de la ST sur la croissance du poulet peuvent être considérées comme sub-optimales, car les doses et(ou) les durées de traitement étaient limitées par les faibles quantités de cST pure disponibles. Avec la fabrication de cST recombinante ( $r-c S T$, Souza et al 1983), qui a une activité bien éta- blie sur la croissance du rat hypophysectomisé (Souza et al 1984, Burke et al 1987, Peebles et al 1988), on a pu enfin essayer de déterminer les effets de l'administration de ST sur les performances du poulet sur des périodes de traitement plus longues. L'administration de fortes doses de r-cST (50, 250 ou $1500 \mu \mathrm{g} / \mathrm{kg} / \mathrm{jour}$ ), trois fois (Burke et al 1987) ou deux fois par jour (Peebles et al 1988) n'a eu aucun effet stimulant sur la croissance de jeunes poulets. Liou et al (1986) ont montré qu'un traitement associant de la r-cST exogène et des hormones thyroïdiennes administrées par voie alimentaire déprime la vitesse de croissance de poulets de chair entre 21 et 42 jours d'âge. Le rendement à l'abattage peut être abaissé chez des oiseaux traités par la r-cST (Cravener et al 1989). Les contenus de la carcasse en protéines et en cendres, ainsi que la rétention azotée ne sont pas modifiés par l'administration de r-cST (Burke et al 1987).

\section{3 / Effets du GRF}

Le GRF stimule la sécrétion de cST aussi bien in vitro que in vivo. Il potentialise aussi la réponse de la sécrétion de cST au TRH (Thyrotropin Releasing Hormone) chez le poulet (Leung et Taylor 1983, Harvey et al 1984, Harvey et Scanes 1985, Leung et al 1985) ou le dindon (Proudman 1984). L'administration de GRF, à des doses connues pour stimuler fortement la sécrétion de cST n'a que des effets transitoires (Leung et al 1986) ou même aucun effet (Buonomo et Baile 1984, 1986) sur le gain de poids et l'efficacité alimentaire de jeunes poulets de chair.

\section{4 / Conclusion}

Johnson (1989) discute des hypothèses qui peuvent être avancées pour expliquer l'absence d'effet de la ST sur les performances des volailles :

- les poulets de chair présentent une croissance initiale très rapide, à un moment où la production endogène de cST est très élevée (Burke et Marks 1982, Vasilatos-Younken et Leach 1986, Vasilatos-Younken et Zarkower 1987),

- les poulets de chair ont un profil de sécrétion de somatotropine particulier, avec des pics de forte amplitude et plus fréquents que dans les autres espèces (Johnson et al 1987 , Vasilatos-Younken et Zarkower 1987). Vasilatos-Younken et al (1988) ont montré que l'administration pulsatile de p-cST améliore les performances de poulettes de 8-11 semaines, alors qu'une infusion continue n'a aucun effet. Ainsi, le profil d'administration de la cST pourrait être important pour obtenir des réponses positives. Ce résultat demande cependant à être confirmé chez des oiseaux plus jeunes.
L'administration de somatotropine ou de GRF ne semble pas avoir d'effet sur les performances $\mathrm{du}$ poulet de chair. 


\section{Conclusion}

L'administration d'hormones somatotropes exogènes ne semble pas avoir d'effet sur les performances et la qualité des carcasses des poulets de chair. Chez le porc, en revanche, elle améliore de façon spectaculaire à la fois la vitesse et l'efficacité de la croissance des tissus maigres et inhibe le dépôt de gras. L'utilisation de GRF ou de pST recombinante amènerait une réduction importante des coûts alimentaires et une amélioration significative de la qualité des carcasses. L'impact du traitement par la pST sur la qualité des viandes semble limité. Les effets négatifs éventuels sur la mise en place de la fonction de reproduction sont transitoires et facilement réversibles après arrêt du traitement. Ainsi, sous réserve que l'on mette au point des systèmes d'administration idoines, le traitement des porcs par les hormones somatotropes exogènes semble être une technique très prometteuse, permettant de mettre sur la marché un produit meilleur marché et mieux adapté à la demande d'une viande maigre. Cependant, la perception par le grand public de l'acceptabilité des viandes provenant d'animaux traités pourrait être une limitation importante à son développement.

Ce texte a été préparé à partir d'un exposé présenté lors du symposium international sur la pST tenu à Washington en décembre 1990

\section{Principales références bibliographiques}

(La liste complète des références citées est disponible auprès de l'auteur)

Bidanel J.P., Bonneau M., Pointillart A., Gruand J., Mourot J. et Demade I., 1991. Effects of exogenous porcine somatotropin (pST) administration on growth performance, carcass traits, and pork meat quality of Meishan, Pietrain, and crossbred gilts. J. Anim. Sci., 69, 3511, 3522 .

Bonneau M., Lefaucheur L. et Mourot J., 1989. Effet de l'administration se somatotropine porcine (pST) sur les performances de croissance, la composition corporelle et la qualité des viandes de porc. Journées Rech. Porcine en France, 21, 31-38.

Bryan K.A., Clark A.M. et Hagen D.R., 1990. Effect of treatment with and subsequent withdrawal of exogenous porcine somatotropin on growth and reproductive characteristics of gilts. J. Anim. Sci., 68, 2357-2361.

Buonomo F.C. et Baile C.A., 1986. Effects of daily injection of growth-hormone-releasing factor and thyrotropin-releasing hormone on growth and endocrine parameters in chickens. Domest. Anim. Endocrinol., 3, 269-276.

Buonomo F.C., Sabacky M.J., Della-Ferra M.A. et Baile C.A., 1987. Effects of somatostatin immunoneutralization on growth and endocrine parameters in chickens. Domest. Anim. Endocrinol., 4, 191-200.

Campbell R.G., Steele N.C., Caperna T.J., McMurtry J.P., Solomon M.B. et Mitchell A.D., 1988. Interrelationships between energy intake and endogenous porcine growth hormone administration on the performance, body composition and protein and energy metabolism of growing pigs weighing 25 to 55 kilograms live weight. J. Anim. Sci., 66, 1643-1655.

Campbell R.G., Steele N.C., Caperna J.P., McMurtry J.P. Solomon M.B. et Mitchell A.D., 1989. The interrelationships between sex and exogenous porcine growth hormone administration on the performance, body composition and protein and fat accretion of growing pigs. J. Anim. Sci., 67, 177-186.

Dubreuil P., Couture Y., Pelletier G., Petitclerc D., Delorme L., Lapierre H., Gaudreau P., Morisset J. et Brazeau P., 1990a. Effect of long-term administration of porcine growth hormonereleasing factor and(or) thyrotropin-releasing factor on growth hormone, prolactin and thyroxine concentrations in growing pigs. J. Anim. Sci., 68, 95-107.

Dubreuil P., Petitclerc D., Pelletier G., Gaudreau P., Farmer C., Mowles T.F., et Brazeau P., 1990b. Effect of dose and frequency of administration of a potent analog of human growth hormonereleasing factor on hormone secretion and growth in pigs. J. Anim. Sci., 68, 1254-1268.
Dubreuil P., Couture Y., Pelletier G., Petitclerc D., Lapierre H., Pommier S., Gaudreau P., Morisset J. et Brazeau P., 1990c. Effect of porcine growth hormone-releasing factor (1-29)NH2 and thyrotropin-releasing factor on pig growth performance. Can. J. Anim. Sci., 70, 459-467.

Etherton T.D., Wiggins J.P., Chung C.S., Evock C.M., Rebhun J.F. et Walton P.E., 1986. Stimulation of pig growth performance by porcine growth hormone and growth hormone-releasing factor. J. Anim. Sci., 63, 1389-1399.

Etherton T.D., Wiggins J.P., Evock C.M., Chung C.S., Rebhun J.F., Walton P.E. et Steele N.C., 1987. Stimulation of pig growth performance by porcine growth hormone : determination of the dose response relationship. J. Anim. Sci., 64, 433-443.

Evock M., Etherton T.D., Chung C.S. et Ivy R.E., 1988. Pituitary porcine growth hormone (pGH) and a recombinant pGH analog stimulate pig growth performance in a similar manner. J. Anim. Sci., 66, 1928-1941.

Goddard C., Wilkie R.S. et Dunn I.C., 1988. The relationship between insulin-like growth factor-1, growth hormone, thyroid hormones and insulin in chickens selected for growth. Domest. Anim. Endocrinol., 5, 165-176.

Harkins M., Boyd R.D. et Bauman D.E., 1989. Efect of recombinant porcine somatotropin on lactational performance and metabolite patterns in sows and growth of nursing pigs. J. Anim. Sci., 67, 1997-2008.

Johnson R.J., 1989. Growth physiology and biotechnology: potential to improve broiler production. World's Poultry Sci. J., $46,228-240$

Kanis E., Nieuwhof G.J., De Greef K.H., van der Hel W., Verstegen M.W.A., Huisman J., et van der Wal P., 1990. Effect of recombinant porcine somatotropin on growth and carcass quality in growing pigs: interactions with genotype, gender and slaughter weight. J. Anim. Sci., 68, 1193-1200.

Kirkwood R.N., Thacker P.A. et Laarveld B., 1989. The influence of exogenous growth hormone on ovulation rate in gilts fed diets with different energy and protein levels. Can. J. Anim. Sci., $69,265-268$.

Louveau I., Bonneau M., Salter D., Prunier A., 1991. Age-related changes in plasma porcine growth hormone (GH) profiles and insulin-like growth factor-I (IGF-I) concentrations in Large White and Meishan pigs. Reprod. Nutr. Dev., 31, 205-216.

Machlin L.J., 1972. Effect of porcine growth hormone on growth and carcass composition of the pig. J. Anim. Sci., 35, 794-800. 
McLaren D.G., Bechtel P.J., Grebner G.L., Novakofski J.E., McKeith F.K., Jones R.W., Dalrymple R.H. et Easter R.A., 1990. Dose response in growth of pigs injected daily with porcine somatotropin from 57 to 103 kilograms. J. Anim. Sci., 68, 640651.

Mourot J., Bonneau M., Charlotin P. et Lefaucheur L., 1990. Effects of exogenous porcine somatotropin (pST) administration on pork meat quality. Meat Science, 31, 219227.

Pommier S.A., Dubreuil P., Pelletier G., Gaudreau P., Mowles T.F., et Brazeau P., 1990. Effect of a potent analog of human growth hormone-releasing factor on carcass composition and quality of crossbred market pigs. J. Anim. Sci., 68, 1291-1298.
Scanes C.G., Duyka D.R., Lauterio T.J., Bowen S.J., Huybrechts L.M., Bacon W.L. et King D.B., 1986. Effects of chicken growth hormone, triiodothyronine and hypophysectomy in growing domestic fowl. Growth, 50, 12-31.

Solomon M.B., Campbell R.G., Steele N.C., Caperna T.J. et McMurtry J.P., 1988. Effect of feed intake and exogenous porcine somatotropin on longissimus muscle fiber characteristics of pigs weighing 55 kilograms live weight. J. Anim. Sci., 66, 32793284 .

Solomon M.B., Campbell R.G. et Steele N.C., 1990. Effect of sex and and exogenous porcine somatotropin on longissimus muscle fiber characteristics of growing pigs. J. Anim. Sci., 68, 11761181.

\section{Summary}

Exogenous administration of GRF or somatotropin in poultry and pigs : effects on performance, meat quality and reproductive function

In ad libitum-fed pigs administered with porcine somatotropin (pST) during the finishing period, daily feed intake is reduced (2-22\%), growth is generally accelerated (up to 47\%) and feed efficiency is dramatically improved $(3-38 \%)$, in connection with a sharp reduction in fat deposition $(7-44 \%)$. Lean content of the carcass is augmented (2-23\%). Due to the increased weight of some organs (liver, heart, etc.), dressing percentage is reduced (1-4\%). Administration of GRF or of a GRF analog has qualitatively the same effects as pST.

The main factors of variation of pST effect on performance and carcass characteristics of pigs include:

1) Dose of pST. Daily feed intake and fat deposition decline linearly with increasing pST doses while the response of feed efficiency, growth rate and lean deposition is quadratic.

2) Mode of administration. Sustained release of pST seems to be less efficient than daily injection.

3) Age and weight. The effects of pST are similar, both qualitatively and quantitatively, during the growing period or in heavy pigs than during the finishing period.

4) Genotype, sex and castration. The effects of pST are negatively related to the animal's potential for lean tissue growth.
The effect of pST on the percentage of the various muscle fiber types is unclear. However, an increase in their size is most often noticed. PST effects on muscular characteristics may vary widely according to muscles. Physical and sensory properties of meat are generally unaffected or only slightly altered by pST treatment. Besides the pST effects on muscle and fat composition, the most consistent changes concern elevated ultimate $\mathrm{pH}$ measurements, increased shear force values and decreased tenderness. Consumer acceptance of fresh pork or processed products does not seem to be impaired by pST treatment.

The impact of pST treatment on the onset of puberty is not clear. However, there is convincing evidence that any possible adverse reproductive change associated with chronic administration of pST to prepubertal gilts is transient. Administration of pST during gestation has no effect on litter. The impact of pST treatment on milk production by lactating sows is still controversial.

Although somatotropin seems to be involved to some extent in the regulation of growth in birds, exogenous administration of GRF or somatotropin has only a transient effect (or, most often, no effect at all) on growth performance and carcass characteristics of chickens.

BONNEAU M., 1992. Administration de GRF ou de somatotropine chez le porc et les volailles : effets sur les performances, la qualité des viandes et la fonction de reproduction. INRA Prod. Anim., 5 (4), 257-267. 